\title{
Der heilige Skarabäus de Else Jerusalem. Un roman entre naturalisme et critique sociale gauchiste
}

Der heilige Skarabäus von Else Jerusalem. Ein Roman zwischen Naturalismus und linker Sozialkritik

The Red House by Else Jerusalem. A Novel between naturalism and left-wing social critique

\section{Sigrid Schmid Bortenschlager}

\section{(Q) OpenEdition}

\section{Journals}

Édition électronique

URL : http://journals.openedition.org/austriaca/578

DOI : 10.4000 /austriaca. 578

ISSN : 2729-0603

\section{Éditeur}

Presses universitaires de Rouen et du Havre

\section{Édition imprimée}

Date de publication : 1 juin 2018

Pagination : 117-133

ISBN : 979-10-240-1233-9

ISSN : 0396-4590

\section{Référence électronique}

Sigrid Schmid Bortenschlager, «Der heilige Skarabäus de Else Jerusalem. Un roman entre naturalisme et critique sociale gauchiste », Austriaca [En ligne], 86 | 2018, mis en ligne le 01 juillet 2020, consulté le 29 janvier 2021. URL : http://journals.openedition.org/austriaca/578 ; DOI : https://doi.org/10.4000/ austriaca. 578 


\title{
Der heilige Skarabäus de Else Jerusalem
}

\author{
UN ROMAN ENTRE NATURALISME \\ ET CRITIQUE SOCIALE GAUCHISTE
}

\section{Un roman à grand succès}

Le «Nagl-Zeidler-Castle», ce trésor positiviste de la littérature autrichienne ${ }^{1}$, voit dans le roman Der heilige Skarabäus d'Else Jerusalem un exemple du naturalisme tardif en Autriche ${ }^{2}$.

Ce « roman sur un bordel» fut l'un des grands succès de l'année 1908, il connut de multiples rééditions successives (je possède une $23^{\mathrm{e}}$ édition de 1911) et des traductions en russe (1910), néerlandais (1911), français (1912), hongrois (1919), letton (1931) et anglais (1932), toutes étant des versions raccourcies ${ }^{3}$. En 1928, il fut adapté pour le cinéma muet ${ }^{4}$. Le film, de la même façon que le roman, provoqua un scandale et fut interdit ${ }^{5}$. Après un recours, la projection en fut autorisée, mais il resta interdit aux mineurs. Le livre comme le film furent interdits

1. Deutsch-österreichische Literaturgeschichte. Ein Handbuch zur Geschichte der deutschen Dichtung in Österreich-Ungarn, Johann W. Nagl, Jakob Zeidler et Eduard Castle, Wien, Fromme, 1899-1937, 4 vol., t. IV, p. 2221 et suiv.

2. Else Jerusalem, Der heilige Skarabäus. Roman, [1909], Brigitte Spreitzer (éd.), Wien, $\mathrm{dvb}$, Das vergessene Buch, 2016. Les $\mathrm{n}^{\mathrm{os}}$ de page entre parenthèses dans le texte renvoient à cette édition.

3. Ibid., p. 609.

4. Die Rothausgasse, Deutschland, 1928, réalisateur: Richard Oswald; acteurs et actrices: Grete Mosheim, Gustav Fröhlich Hilde Jennings, Else Heims.

5. Le sujet du film est le triangle Milada-Horner-Gustav Brenner; ni le début - l'histoire de Katerine - ni la fin - la maison dans la montagne - n'y figurent. À la fin du film, Milada s'en sort et commence une nouvelle vie avec Gustav. La censure critique le fait que le salon soit représenté comme une sorte de pensionnat ( der Salon als eine Art Pensionat dargestellt wird», http://www.difarchiv.deutschesfilminstitut.de/filme/foo1113.htm). 
par les nazis, de même que toutes les œuvres d'Else Jerusalem, qui habitait en Argentine depuis 1911. Dans les années 1980, le roman fut redécouvert à la faveur de recherches sur les écrivaines oubliées ${ }^{6}$. On le retrouve mentionné de temps en temps sous l'étiquette de "roman de prostitution », une définition à la fois correcte et fausse. Il se déroule certes dans un bordel à Vienne, mais ceux qui cherchent de l'érotisme ou de la pornographie seront déçus ${ }^{7}$. On ne peut le comparer ni avec Josefine Mutzenbacher, Der Roman einer Wiener Dirne ${ }^{8}$, de la même période, ni avec Nana de Zola, même si des comparaisons sont faites dans les critiques contemporaines. Rien à voir non plus avec La Dame aux Camélias, roman mentionné dans le texte, ni avec cette tradition de la femme fatale comme figure littéraire telle qu'elle apparaît dèjà dans le roman Manon Lescaut de l'abbé Prévost.

La première partie du roman, "Die schwarze Katerine », fut à plusieurs reprises comparée au Tagebuch einer Verlorenen de Margarete Böhme ${ }^{9}$. On peut aussi penser à Die freudlose Gasse de Hugo Bettauer ${ }^{10}$, mais ce genre de biographie tragique d'une prostituée constitue seulement une petite partie du roman, qui transcende les définitions du naturalisme, comme par exemple la découverte littéraire de nouveaux sujets sur les opprimés - on pense ici au célèbre drame Die Weber (Les Tisserands) de Gerhart Hauptmann. Le livre de Jerusalem inclut, bien sûr, la description de la misère des prostituées, mais sa spécificité

6. On trouve facilement les articles dans le catalogue de l'Österreichischen Nationalbibliothek, surtout sur son site «Ariadne»

7. Mais Vienne n'est jamais mentionnée directement, au contraire, on a essayé d'en faire un mélange international - le Louvre se trouve près de la Kärtnerstrasse, l'hôpital central est dans la Luisenstrasse, comme à Berlin, etc. Mais les lieux d'origine des prostituées et surtout leurs accents, qui sont rendus précisément dans les dialogues, pointent clairement qu'il s'agit de Vienne.

8. Anonym (Felix Salten), Josefine Mutzenbacher, oder Die Geschichte einer Wienerischen Dirne von ihr selbst erzählt. Ungekürzter Nachdruck der Ausgabe von Wien, 1906, München, Schneekluth, 1990 [Josefine Mutzenbacher. Histoire d'une fille de Vienne racontée par elle-même, Paris, Mercure de France, 1979; rééd. : Paris, Gallimard, «Folio, 3057", 1998].

9. Voir par exemple Eva Borst, «Ichlosigkeit als Paradigma weiblichen Daseins. Prostitution bei Margarete Böhme und Else Jerusalem », dans Karin Tebben (dir.), Deutschsprachige Schriftstellerinnen des Fin de Siècle, Darmstadt, Wissenschaftliche Buchgesellschaft, 1999, p. 114-137. Tagebuch einer Verlorenen, von einer Toten. Überarbeitet und hg. von Margarete Böhme, Berlin, Fontane, 1905.

10. Hugo Bettauer, Die freudlose Gasse. Ein Wiener Roman aus unseren Tagen, Wien, Leipzig, Löwit, 1924. Le livre est devenu célèbre grâce au film avec Greta Garbo et Asta Nielson. 
est d'analyser le fonctionnement du bordel en le représentant comme une entreprise capitaliste, tout en évitant les questions morales ou moralisantes. La perspective d'un enfant, Milada, né et grandissant dans un bordel, donne un caractère «naturel » à cette partie spécifique de la société.

Construction littéraire complexe, le roman est écrit du point de vue d'un narrateur omniscient. Si on se réfère au strict sens littéraire, ce n'est pas le point de vue de Milada qui domine. Le texte comporte quelques passages un peu pathétiques. Mais ce qui frappe, c'est l'absence de notions religieuses telles que le péché, la faute, la pénitence, le châtiment, ainsi que celles de jouissance, de sexualité, d'érotisme. La narratrice se concentre sur les aspects économiques et fonctionnels. La seule chose qui intéresse vraiment les propriétaires successives de la maison close est le rendement de plus de cent pour cent sur le capital investi. Une fois le capital souhaité atteint, les deux premières propriétaires se consacrent à leurs vies privées. $\mathrm{M}^{\mathrm{me}}$ Goldscheider devient une mère riche, capable de donner une éducation chrétienne et bourgeoise à sa fille adorée. $\mathrm{M}^{\text {lle }}$ Miller, quant à elle, peut finir ses jours comme chanoinesse respectée dans un couvent.

\section{La discussion publique sur la prostitution}

La prostitution était un sujet sensible à la fin du XIX ${ }^{\mathrm{e}}$ siècle, débattu dans les journaux et au sein des corps législatifs et juridiques. Les mouvements d'émancipation des femmes furent aussi confrontés au problème. Le débat se déroulait sur trois axes: la morale (invoquée par les milieux religieux et les partis conservateurs), l'émancipation des femmes (allant chez les radicaux comme Troll-Borostyáni ${ }^{11}$ jusqu’à la comparaison de la prostitution au mariage, les deux seules issues pour les femmes, étant donné qu'elles ne peuvent pas gagner une vie digne par elles-mêmes) et la santé publique (surtout au sein des partis socialistes), c'est-à-dire le danger de la syphilis, maladie très répandue et inguérissable à l'époque. Au sein de ces débats, les solutions proposées étaient assez hypocrites et diverses, tout comme aujourd'hui.

On a reproché à Else Jerusalem de proposer une solution idéaliste, l'héroïne, Milada, fondant à la fin du roman un établissement pour les

11. Irma von Troll-Borostyáni, Die Prostitution vor dem Gesetz. Ein Appell ans deutsche Volk und seine Vertreter, Leipzig, Clausner, 1893. 
enfants des prostituées. Même si le but des romans n'est pas de proposer des solutions à des problèmes sociaux, ces reproches ne tiennent pas compte du fait que différents remèdes sont envisagés, puis écartés dans le livre: l'organisation syndicale des prostituées (proposée par Horner), l'exaltation de la prostitution (des femmes, bien sûr) comme libération de la société (du même Horner); le sauvetage de certaines prostituées par des bourgeois engagés (la pauvre paysanne, la jeune atteinte de tuberculose, Fritzi de Brünn avec son amant officier). Ces efforts de rédemption ne sont pas acceptés par les filles elles-mêmes, parce qu'elles savent que la société n'accepterait jamais le retour d'une prostituée à une vie «normale», soit villageoise, soit bourgeoise. Même dans les cas où le droit est tout à fait du côté de la prostituée, la corruption de la police et de la justice garantit le maintien de l'ordre public tel qu'il est accepté de tous.

La solution proposée à la fin du livre - certainement assez idéaliste et romanesque - est mûrement réfléchie. Il $\mathrm{n}$ y a pas de solution sociale, l'héroïne ne peut compter que sur ses propres moyens, ou, pour citer Candide de Voltaire : "Il faut cultiver notre jardin. »

Pour excuser l'héroïne et, plus encore, l'écrivaine, il faut souligner que la solution au problème de la prostitution n'a pas vraiment progressé depuis l'époque du roman jusqu'à nos jours. Le livre décrit les deux formes qui existent autour de 1900 : la prostitution de rue et celle des maisons closes. La situation légale était claire et ambiguë à la fois. À Vienne, où se déroule le roman, la prostitution était prohibée, mais, dès 1873 , le Büchel (littéralement: «livret»), fut introduit ${ }^{12}$. Il fallait $\mathrm{y}$ consigner les résultats d'un contrôle médical à réaliser deux fois par semaine, aux frais de la prostituée. Mais la prohibition totale de la prostitution n'en était pas pour autant levée, ce qui a causé cette situation ambiguë: les prostituées munies de ce carnet de santé étaient tolérées, sauf si elles avaient causé "un trouble à l'ordre public», ce qui restait à définir. C'était exactement ce «sauf si » qui les rendaient vulnérables. L'atteinte à l'ordre public était vite établie, le fait de chercher des clients pouvant suffire. Il était même impossible de forcer les clients à payer:

12. En octobre 1874,6422 «livrets» furent délivrés; on estime le nombre de prostituées illégales à 12000 - pour une ville de 2 millions d'habitants. Pour comparer les chiffres avec ceux de nos jours, en 2013, Vienne, qui a à peu près la même taille, compte, officiellement, environ la moitié de prostitués seulement, soit 3390 femmes et 67 hommes. 
le service rendu étant illégal, aucun contrat valable ne pouvait être invoqué ${ }^{13}$.

Les féministes, avec les abolitionnistes, se sont pour la plupart prononcées pour la libéralisation totale de la prostitution, en en faisant un métier comme les autres. Les conservateurs - et beaucoup de libéraux - ont préféré la mettre hors de vue, dans des bordels. Les arguments avancés étaient la nécessité de sécuriser les rues de la ville, de protéger les enfants et les femmes respectables du spectacle des prostituées et d'exercer un meilleur contrôle médical.

\section{Prostitution de rue vs maisons closes}

Dans la première partie de son roman, Jerusalem décrit ce changement de la prostitution de rue à la prostitution dans le bordel. Katerine commence comme prostituée libre, comme Freimädel ${ }^{14}$, elle a son « livret», mais, parallèlement, elle mène une vie «normale». Elle a un petit appartement avec sa fille et son amie Janka qui s'occupe de la maison; elle travaille comme prostituée, mais c'est elle qui choisit ses clients, et il peut arriver qu'elle tombe amoureuse d'un garçon et le prenne gratuitement comme amant. Il y a même des périodes où elle ne travaille pas, parce qu'elle a gagné assez d'argent pour vivre. Quand "La Maison rouge», où elle habite, est transformée en maison close, elle peut y rester. C'est alors qu'elle change son nom en Carmen, pour être plus originale. C'est aussi à ce moment-là qu'elle arrête de croquer des pommes et les remplace par du vin - l'auteure signalant ainsi la dépersonnalisation que le bordel produit.

13. Ce n'est pas nécessaire de se moquer de l'hypocrisie et de l'inconséquence de l'attitude envers la prostitution au XIX ${ }^{\mathrm{e}}$ siècle. Aujourd'hui, en Allemagne et en Autriche, la prostitution est libre, c'est un métier comme les autres, soumis à l'impôt, la sécurité sociale, etc. En France, au contraire (comme en Suède, pour démontrer qu'il ne s'agit pas d'une question de "progressisme»), la prostitution est complètement interdite, avec des sanctions pour les deux parties.

14. Katerine tombe enceinte d'un agriculteur bohémien très riche, qui n'a jamais eu l'intention d'épouser cette séduisante jeune femme pauvre. Il lui offre une compensation monétaire, mais pour elle, le reste de sa vie est consacré à la vengeance. Elle devient officiellement ce que, lui, a fait d'elle - une prostituée. Elle va à Vienne avec son amie, la nièce de l'agriculteur, donne naissance à une fille, et toutes les trois vivent la vie des prostituées libres (Freimädel); quand leur maison est transformée en bordel, Janka retourne dans sa famille, Katerine et Milada y restent. 
L'histoire de Katerine s'inscrit dans le genre de la biographie tragique des prostituées. Au début, elle représente le type de la putain selon Weininger ${ }^{15}$, et non celui de la mère, bien que ce soit elle la mère biologique de Milada - mais rien de plus. C'est Janka, la vraie femme mère. La vitalité de Katerine et l'énergie que l'idée de la vengeance lui donne déclinent lentement; après quatorze années dans le métier, elle tombe malade et doit quitter le bordel pour la province. L'alcool l'a détruite et elle meurt à l'hospice.

En parallèle du lent déclin de l'héroïne Katerine, s'opère la lente ascension de "La Maison rouge ». L'électricité et le tout-à-l'égout arrivent, la ruelle est pavée, des règles sanitaires sont imposées, et, à cause de tout cela, la majorité des prostituées est forcée de ne plus exercer ici et de quitter cette ruelle. Ce que le lecteur ou la lectrice interprète comme un exemple de l'amélioration des conditions de vie des grandes métropoles se trouve contredit quelques pages plus loin. Il s'agit en réalité d'une manœuvre du chef de la police des mœurs dans une complexe affaire immobilière. Cet homme veut vendre "La Maison rouge", avec une licence de bordel. Mais pour parvenir à ses fins, il doit d'abord rendre l'affaire plus intéressante.

\section{Le salon Goldscheider}

Ce qui importe ici, c'est que $\mathrm{M}^{\mathrm{me}}$ Goldscheider n'est pas du tout une "Madame », une «mère-maquerelle». Au contraire, c'est une veuve juive plutôt traditionnelle, qui gère des commerces de vêtements. Elle, qui est d'origine très modeste, pauvre même, a une seule passion dans la vie : sa fille, qu'elle veut éduquer comme une bourgeoise protestante pour lui ouvrir toutes les possibilités du monde. Pour cela, il lui faut beaucoup d'argent, et le profit de plus de cent pour cent qu'on lui a promis lui fait prendre l'affaire en considération. En bonne commerçante, elle fait le tour des bordels d'Autriche-Hongrie et d'Allemagne pour voir si les promesses de rendement sont avérées et pour se familiariser avec le métier. Son tour d'horizon se révélant satisfaisant, elle achète « La Maison rouge».

Elle sait qu'il faut lui investir pour gagner de l'argent. Elle gère la maison et les filles d'une manière généreuse, il y a des salons confortables, des repas et des alcools excellents, et des filles jolies, plaisantes et gaies

15. Otto Weininger, Geschlecht und Charakter, Wien, Braumüller, 1902. 
qui changent souvent. Il y a même des bourgeoises qui gagnent ici, de temps en temps, un peu d'argent de poche. $\mathrm{M}^{\text {me }}$ Goldscheider est donc le contraire de $\mathrm{M}^{\text {me }}$ Riehl, dont le procès à Vienne en 1906 (il commence le 2 novembre) est souvent cité comme source du roman ${ }^{16}$. Else Jerusalem fut l'une des deux femmes à suivre le procès pour la Frauenliga gegen den Mädchenhandel (la Ligue des femmes contre le trafic des filles) ${ }^{17}$.

Le bordel de $\mathrm{M}^{\mathrm{me}}$ Goldscheider est plutôt un établissement propre et sérieux, mais l'histoire de Katerine montre que la perte de la liberté du Freimädel conduit à une perte de confiance en soi, même dans le meilleur des cas. Dans la maison close, tout est arrangé pour les filles: elles ont leur chambre, leurs repas, leurs vêtements, leurs horaires de travail, les clients viennent jusqu'à elles. Elles apprécient leurs conditions de vie, surtout si elles considèrent les alternatives: le dur labeur physique à la campagne, dix à douze heures de travail, six jours par semaine, en usine, le travail comme domestique avec un seul dimanche libre par mois, dans tous les trois cas très mal payés. Mais elles deviennent lasses, perdent leur vitalité et en viennent finalement à accepter d'être envoyées vers d'autres établissements, sans avoir leur mot à dire.

Et c'est bien ce trafic humain qui génère une bonne partie de l'argent dans ce métier. Cela commence par le recrutement. Les agents - dans le roman ce sont exclusivement des femmes - arpentent les grandes gares de la capitale où débarquent les jeunes filles arrivant de la campagne à la recherche d'un travail en ville. Les recruteuses s'occupent d'elles, les invitent à un repas, leur donnent un lit pour quelques jours et leur vantent les avantages de travailler dans une maison close, l'argent qu'on peut facilement y gagner, la vie de luxe, etc.

Le deuxième lieu d'enrôlement qui marche encore mieux, ce sont les maternités. C'est là qu'échouent les jeunes domestiques qui se sont retrouvées enceintes et ont perdu leur emploi. Elles n'ont alors plus

16. Le procès Riehl est connu aujourd'hui principalement à cause de Karl Kraus, qui lui a consacré un numéro de Die Fackel ( $\mathrm{n}^{\circ}$ 211, novembre 1906) qui est d'une misogynie étonnante.

17. Voir Brigitte Spreitzer dans Der heilige Skarabäus, op. cit., p. 553. On reviendra à cette question. Il faut dire que c'était un travail formidable que d'écrire un roman de 686 pages, de trouver un éditeur et de le publier en deux ans et demi - le roman est paru en juin ou juillet 1909. Pour ma part, je pense que le roman était déjà bien avancé quand le procès a commencé - c'est seulement dans le chapitre «Das System Spizzari» que l'on trouve de vrais détails mentionnés dans le procès - et ils ne sont pas très bien intégrés dans la totalité du roman. Le thème de la prostitution se trouve déjà dans la première publication de Jerusalem, en ce temps-là encore appelée Kotanyi, Venus am Kreuz. Drei Novellen, Leipzig-Berlin, Meyer, 1899. 
aucune solution. Les agents offrent de prendre à leur charge le coût de l'hôpital et de trouver une place pour le bébé si elles consentent à travailler comme prostituées. Les jeunes femmes commencent donc leur travail en ayant contracté des dettes considérables, que la plupart d'entre elles n'arrivent jamais à rembourser, même avec des directrices honorables comme $\mathrm{M}^{\text {me }}$ Goldscheider ${ }^{18}$.

Mais le travail au bordel peut rapporter aussi pas mal d'argent, comme le montre l'exemple de la petite Bine qui envoie en un an plus de 800 gulden à sa supposée future belle-mère (p. 243). L'histoire de Bine est l'un des nombreux intermèdes du roman. La jeune campagnarde est promise comme fiancée à un voisin qui fait son service militaire. Pour gagner l'argent de leur future vie commune, elle se rend en ville et travaille dans "La Maison rouge», en toute innocence. Mais, bien sûr, la future belle-mère, après avoir accepté l'argent de Bine, trouve une autre femme pour son fils. En retournant au village pour une fête, Bine se trouve confrontée à la cruelle réalité, qu'elle accepte après le choc initial. Une fille avec un passé comme le sien n'a plus aucun avenir au village: «Braves [sic] Mann kann so eine nicht mehr heiraten... Wär schad um ihn» (p. 309). Sans protester et sans poser de questions, elle quitte «La Maison rouge " pour un autre établissement de province, comme le font toutes les autres filles après un certain temps.

Il y a aussi la petite Fanny Fanchon (p. 254 et suiv.), jeune prolétarienne, qui a mangé sa première viande à 13 ans, des saucisses de Francfort. Ce sont des détails concrets de cette sorte qui permettent de parler de naturalisme. Pour Fanny, «La Maison rouge» représente presque le paradis. Quand elle tombe malade, elle est soignée par Gust Brenner. Quand celui-ci - poussé par Milada - lui propose de faire soigner sa tuberculose dans un hospice financé par son père, à la campagne, elle refuse violemment et l'accuse de l'avoir infectée et de vouloir la tuer pour cacher ses erreurs.

Pour la plupart des prostituées décrites dans le roman, la vie dans « La Maison rouge » est la meilleure chose qui leur soit arrivée, même si elles ne parviennent pas à vraiment accumuler de l'argent. En comparaison de la vie des ouvrières dans les usines ou de celle des domestiques, elles mènent une vie de petit luxe sans souci. Mais les comptes de $\mathrm{M}^{\mathrm{me}}$ Goldscheider ne leur sont presque jamais favorables. Leurs dettes sont exigibles seulement lorsqu'elles quittent l'établissement. Elles n'ont

18. Beaucoup ont aussi des obligations envers des membres de leur famille, des parents malades, des frères endettés, etc. 
aucune autre possibilité que d'accepter sans protestation d'être envoyées - ou plutôt vendues - à d'autres établissements, d'un niveau inférieur. Le prix mentionné par la petite Fanchon est - malgré sa tuberculose de 500 gulden (p. 276).

\section{Les raisons de devenir prostituée}

C'est Milada qui explique à Gust l'existence de la prostitution, d'une part, comme résultat de la forte demande, et, d'autre part, comme la conséquence des conditions sociales catastrophiques des jeunes filles - faim et désespoir («Hunger und Verzweiflung», p. 265). Plus tard, lorsque tous deux parlent encore une fois de ce sujet - Gust Brenner essaie d'extraire Milada de «ce milieu »-, elle concède qu'il y a aussi des filles qui sont insouciantes par nature ("Leichtsinn im Blute», p. 347), bien qu'il ne soit pas juste que la société leur facilite autant et de façon irréversible la possibilité de devenir prostituées.

Jerusalem s'insère dans la discussion sur l'hérédité de la pulsion sexuelle chez la femme, une question qui l'a déjà intéressée dans son premier livre, Venus am Kreuz, où la protagoniste est la fille d'une maîtresse qui, malgré ses efforts pour vivre une vie normale, finit comme prostituée. C'est aussi le sujet du célèbre livre d'Otto Weininger, Geschlecht und Charakter. Pour lui, les femmes sont, par nature, soit mères soit putes. Il n'y a pas de choix individuel possible. Mais, dans Der heilige Skarabäus, Jerusalem se détermine contre l'hérédité et prend une position clairement socialiste. Milada, pourtant fille d'une prostituée, parvient à s'éduquer, bien qu'elle grandisse dans un bordel. La lecture et le travail lui offrent un chemin vers la réalisation de soi, comme on dirait aujourd'hui.

C'est avec surprise que Gust Brenner découvre dans la chambre de Milada les livres qu'elle a bel et bien feuilletés, "Marx, Le Capital, ... L'Unique et sa propriété [Stirner] - Parerga und Paralipomena [Schopenhauer] - Force et matière [Ludwig Büchner] - Bebel - Fournier - il eut le vertige» ( "Marx, Das Kapital... Der Einzige und sein Eigentum - Parerga und Paralipomena - Kraft und Stoff - Bebel - Fournier - ihm schwindelte», p. 263). Ce sont les livres que Horner, son maître, lui a donnés, mais c'est aussi la sélection qu'elle a faite elle-même, parce que la philosophie proposée par Horner est très éclectique et mixte, et contient certainement aussi pas mal d'ouvrages de Nietzsche ainsi que quelques anarchistes. Mais la liste citée représente aussi la position de Jerusalem 
elle-même. Elle fait partie à Vienne du cercle des intellectuels de gauche, comme l'indique sa conférence "Gebt uns die Wahrheit! ${ }^{19}$ " dans la célèbre Wiener Volkshochschule, l'université populaire fondée par des socialistes et qui existe toujours. On y trouve Mahler, Broch, Canetti, etc. comme enseignants ${ }^{20}$.

La grande majorité des exemples donnés dans le roman expliquent le sort des prostituées par leur misère sociale. Parmi elles, Katerine, la mère de Milada, constitue une exception. Elle refuse la compensation monétaire offerte par son amant et préfère vivre sa vengeance. Elle est violée dans son amour propre et choisit consciemment le chemin de la destruction. Dans le dernier chapitre, on trouve aussi une femme bourgeoise qui fréquente volontairement des bordels pour y trouver des partenaires sexuels (p. 483 et suiv. ${ }^{21}$, mais elle est clairement désignée comme «malade» et méprisée par les autres.

Dans le roman de Jerusalem, la prostitution n'est donc ni le résultat de l'hérédité, ni du milieu, mais de l'organisation de la société, qui n'offre à bien des jeunes femmes d'autres possibilités de gagner leur vie.

Le roman est sur ce point conforme aux théories du naturalisme qui veulent que la société soit expliquée au travers d'un prisme scientifique - dans le cas présent, c'est le marxisme qui offre le cadre à la narratrice, et non pas le darwinisme, l'eugénisme ou la psychanalyse, tous plus populaires. Le marxisme est en train de s'établir non pas comme nouveau paradigme dans l'économie ou dans l'histoire, mais dans l'arène politique. En Autriche, l'origine de la Sozialdemokratische Arbeiterpartei date de 1889; en 1907, après l'introduction du suffrage universel (pour les hommes seulement, bien sûr), il devient le deuxième parti au Reichstag.

19. Else Jerusalem, Gebt uns die Wahrheit! Ein Beitrag zu unserer Erziehung zur Ehe, Leipzig, Seemann, 1902.

20. Ce milieu de la gauche antifasciste des années vingt est aussi le milieu que fréquente Jerusalem quand elle voyage en Europe. Donc, contrairement à ce que sa petite-fille écrit dans la préface à la nouvelle édition de Skarabäus, Jerusalem, vraisemblablement, n'était pas déçue par son fils Wilhelm, issu de son premier mariage, qui se battit lors de la guerre civile en Autriche, puis en Espagne et en Chine, y devenant le correspondant de la Volksstimme, l'équivalent autrichien de L'Humanité. Au contraire, on peut supposer qu'elle était fière de sa position antifasciste vécue de l'intérieur; seul son communisme avéré n'était probablement pas totalement à son goût.

21. "Durch und durch von Krankheit verwüstet, war sie eine jener tiefbeklagenswerten Frauen, die ein dunkler, nimmersatter Trieb zur Dirnemacht » (p. 483 et suiv.). 
L'analyse de la prostitution par la narratrice du roman suit les idées marxistes, comme Milada l'explique à Gust (p. 265 et suiv.). La prostitution existe à cause de la demande. C'est une entreprise comme une autre: les travailleuses y succombent parce que, pour la plupart d'entre elles, il ne leur reste plus aucune autre possibilité, après avoir été exploitées comme domestiques, surtout si elles sont tombées enceintes. Il est vrai que le bordel, s'il est bien géré, offre quelques années d'une vie plutôt acceptable. Pourtant, un jour ou l'autre, la descente inéluctable commence, mais ce sont quand même quelques années convenables de gagnées. Gust parle de la responsabilité de chacun et de chacune, des sacrifices qu'il faut faire, de la morale - mais il est évident que lui, jeune homme gâté, n'y comprend rien.

Milada, qui connaît le milieu comme personne d'autre, a certainement pensé à la solution socialiste, l'organisation des prostituées, mais elle sait que cela ne marchera jamais ; même cent ans plus tard, ce n'est pas chose gagnée. Elle décidé donc de faire ce qu'elle peut - gérer le bordel où elle travaille d'une manière propre et honnête, et accumuler assez d'argent pour entretenir une maison pour les enfants des prostituées.

\section{Le salon Miller}

La bonne gestion devient une chose difficile quand $\mathrm{M}^{\text {me }}$ Goldscheider vend la maison à $\mathrm{M}^{\text {lle }}$ Miller. L'anticléricalisme manifeste dans l'histoire de $\mathrm{M}^{\text {lle }}$ Miller est un autre signe de l'appartenance de Jerusalem aux milieux gauchistes. $\mathrm{M}^{\text {lle }}$ Miller était la bonne d'un curé de campagne; elle gère la maison et la paroisse avec sévérité et une avarice prononcée, mais après la mort du curé, il ne lui reste pas assez d'argent pour finir sa vie confortablement. Elle se souvient alors d'un ancien conseiller et lui demande comment elle pourrait investir son capital. Il lui propose d'acheter le bordel de $\mathrm{M}^{\text {me }}$ Goldscheider. $\mathrm{M}^{\text {lle }}$ Miller est dans un premier temps légitimement choquée, mais quand elle entrevoit les rendements possibles, elle se laisse vite convaincre, l'achète et commence à le gérer elle-même. Contrairement à l'ancienne propriétaire, elle n'a aucune expérience et essaie de faire des économies dans tous les domaines et arrive presque à ruiner l'établissement. C'est à ce moment-là que Milada devient active. Elle l'aide dans ses relations avec la police, les filles, les livreurs et réussit à restaurer le niveau du bordel et à en faire de nouveau une entreprise rentable. $\mathrm{M}^{\text {lle }}$ Miller devient vraiment dépendante d'elle, et Milada arrive à se faire payer à hauteur d'un tiers 
des profits. Après quelques années, $\mathrm{M}^{\text {lle }}$ Miller parvient à son but : ayant accumulé assez de capital pour devenir chanoinesse, elle vend alors le bordel à $\mathrm{M}^{\mathrm{me}}$ Spizzari.

L'idée de faire de la bonne d'un curé de campagne la gérante d'une maison close ne relève ni du réalisme ni du naturalisme, il s'agit plutôt d'une forme amusante de cynisme anticlérical. On le constate aussi dans d'autres passages: Janka essaie de trouver pour Milada une place dans une école catholique, mais elle est refusée par crainte d'une mauvaise influence sur les autres élèves (p. 44 et suiv.). Quelques décennies plus tard, c'est Milada qui se retrouve dans le même cloître pour arranger la position de chanoinesse de $\mathrm{M}^{\text {lle }}$ Miller. Sa réponse à la question relative à «dernière activité » est «tenancière d'une maison close " («Besitzerin eines Freudenhauses Rothausgasse $2 »)$, ce que la sœur traduit pour les documents par "Propriétaire d'un pensionnat agréé par la ville» ( Inhaberin eines städtisch konzessionierten Pensionats», p. 415). Plus tard, Milada change l'inscription sur la maison pour les enfants des prostituées: «Par sa propre force, à la gloire de Dieu » («Aus eigener Kraft, zu Gottes Ehr») devient "Par sa propre force, pour sa propre gloire " ("Aus eigener Kraft, zu eigener Ehr») : "Ainsi avait-elle réglé pour l'éternité la dette des enfants qui seront placés dans ce foyer de la charité» ("Damit hatte sie die Dankesschuld künftiger Pfleglinge dieses Hauses der Wohlfahrt für ewige Zeiten getilgt », p. 525).

\section{Les immigrés juifs}

Les études critiques consacrées au roman se concentrent sur le milieu du bordel et sur l'héroïne Milada, en oubliant qu'y sont dépeints une autre femme forte et un autre milieu défavorisé : $\mathrm{M}^{\mathrm{me}}$ Goldscheider et son enfance dans une famille d'immigrés juifs de Galicie. La famille de sept enfants habite deux chambres en sous-sol avec six sous-locataires, et tous vivent exclusivement d'aumônes; les jeunes hommes passent leur temps entre prières et discussions talmudiques. L'histoire sociale démontre que des conditions comme celles qui sont décrites ici ne sont pas exagérées, mais correspondent à la réalité à Vienne (et dans d'autres grandes villes), dans les dernières années du $\mathrm{XIX}^{\mathrm{e}}$ siècle. C'est Elise Goldscheider qui, à 16 ans, a la responsabilité de nourrir cette tribu, la mère étant trop malade pour le faire. Et c'est elle, guidée par l'attention de son futur mari et la richesse, certainement relative, qu'elle aperçoit dans sa maison, qui change les choses: elle ne demande plus 
d'aumônes, mais du travail, et elle pousse les hommes à travailler. Sa future belle-mère voit la lente métamorphose du ménage et accepte finalement le mariage de son fils avec Elise, à condition que le reste de la famille, sous-locataires inclus, retourne en Galicie (p. 121 et suiv.).

Le jour du mariage, $\mathrm{M}^{\mathrm{me}}$ Goldscheider senior licencie ses deux employés (p. 129), et les remplace par Elise qui continue à travailler pour sa belle-mère jusqu'à sa mort, sans se plaindre. La seule exception dans sa vie traditionnelle juive est l'éducation de sa fille; même là, elle attend la mort de son mari pour la baptiser et changer son nom. Lorsqu'elle est enfin devenue indépendante, elle rompt avec le judaïsme, devient gérante de "La Maison rouge », et, ayant gagné assez d'argent, elle rejoint sa fille bien aimée pour vivre avec elle la vie d'une riche rentière.

\section{Le travail}

Toutes les deux, Milada et Elise Goldscheider, ont vécu une enfance terrible, dans des milieux extrêmement défavorisés, et toutes les deux s'en sont sorties, sans aide, par leur propre force, leur amour du travail.

Dans le roman, le travail est le moyen de la réalisation de soi, et non une punition pour le péché originel, comme il est écrit dans la Bible et comme le vivent les jeunes juifs galiciens ${ }^{22}$. Cette position est, encore une fois, plutôt marxiste - même si Marx rêve d'une société sans travail, où on irait pêcher le matin et peindre l'après-midi. Dans la réalité de son temps, le travail est un droit et il permet l'épanouissement de l'homme et de la femme. Les deux figures positives du roman ont un but dans leur vie, et le travail est le moyen pour y parvenir; qu'importe le type de travail (même la prostitution, la gérance d'un bordel), si c'est du travail bien fait, cela suffit.

Milada et $\mathrm{M}^{\mathrm{me}}$ Goldscheider représentent la possibilité de la réussite. On pourrait dire que les deux autres propriétaires de "La Maison rouge » ont aussi du succès, mais, chez elles, c'est purement financier : $M^{\text {lle }}$ Miller restera, même dans son couvent, quelqu'un de méchant, et $\mathrm{M}^{\mathrm{me}}$ Spizzari est tout simplement une personne exécrable.

22. Pour faire la comparaison avec aujourd'hui, une fois de plus, c'est aussi le mode de vie d'une partie des Hassidim en Israël, qui préfèrent l'étude de la Thora au travail quotidien et qui sont pris en charge par l'État, qui essaie maintenant de changer cette tradition. 


\section{Le salon Spizzari : réalité et fiction}

Beaucoup de détails de ce dernier chapitre sont inspirés directement du procès de $\mathrm{M}^{\text {me }}$ Riehl : l'incarcération des filles dans seulement deux chambres, sans fenêtre, où elles sont enfermées à clé pendant la journée; les vêtements confisqués pour rendre une évasion impossible, le suicide de Rosine, les enfants forcés à des pratiques sexuelles, le passage soudain de la brutalité à la gentillesse, la dépendance psychique des filles, la corruption de la police, etc. Donc, à première vue il s'agit d'un reportage réaliste, voire naturaliste. Mais la description ne convainc pas. Celle de la vie dans une maison close comme celle de $\mathrm{M}^{\text {me }}$ Goldscheider et de $\mathrm{M}^{\text {lle }}$ Miller paraît plus plausible. L'horreur de la lente descente en l'enfer est abordée de temps en temps, avec le départ de certaines filles, mais elle reste dans l'ombre, en arrière-plan. La corruption de la police choque Gust Brenner, mais pas Milada ni les lecteurs ou les lectrices. C'est inévitablement comme cela: c'est la vie. On peut comprendre que les prostituées vivent leur vie comme les femmes «normales» vivent la leur - en escamotant les parties désagréables. On peut presque s'identifier à elles, à leurs petites joies et leurs bagarres, cela n'a rien d'extraordinaire. Tandis que dans le salon Spizzari, c'est la terreur manifeste, la lectrice ou le lecteur ne comprend pas pourquoi il n'y a pas de révolte ou de suicide collectif.

Je suppose que cette partie fut seulement incorporée à la fin de l'écriture du livre. Le procès Riehl a certainement impressionné l'auteure, et il a aussi augmenté les chances de succès du livre. Toutefois, c'est comme si elle n'avait pas eu assez de temps pour l'intégrer d'une manière convaincante. La vente à $\mathrm{M}^{\mathrm{me}}$ Spizzari était déjà prévue, mais dans les conversations entre elle et Milada, c'est une autre femme qui parle, une femme qui est certainement laide, un peu étrange, mais qui a des ambitions pour un bordel de haute qualité ; la chambre avec bain turc qu'elle a déjà construite, par exemple, provoque la surprise de Milada : "Même l'Empereur pourrait venir ici", répondait Milada pleine de respect. "Je me contenterais aussi de sa clique” - rétorquait la Spizzari » (" „Da kann der Kaiser her, " antwortete Milada dann ehrfürchtig. "Ich nehm auch sei Mischpoche" - erwiderte die Spizzari», p. 328). La différence avec l'établissement décrit dans le dernier chapitre ne pouvait pas être plus grande. 


\section{La construction du roman}

Le chapitre «Das System Spizzari » est nécessaire à la construction du roman et à son histoire. Le livre commence avec une pseudo-famille composée de deux femmes et un enfant, Katerine, Janka et Milada. Toutes les trois entament leur lente descente. À la fin du roman, nous trouvons un autre ménage à trois: Milada, la jeune Jultsch, enceinte de Gust, le grand amour de Milada, et celui-ci. Cette fois, le ménage repose sur de bonnes bases économiques, Milada est la propriétaire de la maison "Zur lichtigen Höh», située à la campagne, et si elle n'est pas vraiment riche, elle vit au moins avec aisance. En plus, elle a un projet pour le futur, cet asile pour les enfants des prostituées, l'enfant de Jultsch et Gust sera le début d'une grande famille, c'est une ascension qui commence.

On y retrouve encore une fois l'opposition établie par Weininger entre mère et prostituée: Milada, à cause de son métier, a perdu la possibilité d'avoir des enfants et accepte immédiatement l'enfant de Gust et de Jultsch comme son propre enfant; Jultsch, la petite campagnarde un peu stupide, que Gust a sélectionnée pour exercer sa vengeance envers Milada, est faite pour être une mère biologique, mais pas plus, elle est et restera probablement une enfant.

Une autre différence importante est que Milada n'envisage pas d'informer Gust de sa paternité ; elle a été amenée à découvrir son caractère de jeune homme gâté, pas vraiment pourri, mais déformé et corrompu par sa société, membre de la jeunesse dorée de la fin de siècle, sans responsabilité ni loyauté. Elle est contente d'avoir l'enfant, cela lui suffit.

La fin du roman dans la campagne évoque le Heimatroman contemporain, avec l'opposition entre la ville corrompue et la campagne et les montagnes où les valeurs traditionnelles sont conservées. Mais ce n'est certainement pas dans cette tradition qu'on peut insérer le roman, il s'inscrit plutôt dans la critique naturaliste de la campagne que l'on peut trouver chez Schönherr ou Anzengruber. La petite maison "Zur lichtigen Höh» (p. 456 et suiv.) se trouve hors du village, en altitude, dans les pâturages d'été, et est utilisée comme une sorte d'asile pour les femmes pauvres qui ne peuvent plus travailler. La liaison avec le village est entretenue par le fils de la Fattinger Rosl, qui avait douze enfants hors mariage, chacun d'un autre père ("die zwölfuneheliche Kinder hatte und a jeds von an anderen Vatta», p. 456) - la prostitution n'est pas inconnue à la campagne. Il faut aussi penser aux diverses histoires de prostituées venant de la campagne, comme Bine, à qui on vole tout l'argent ainsi que son fiancé, ou comme Jultsch, qui est pratiquement 
vendue au bordel par son propre père, pour constater que la campagne n'est pas décrite comme moralement meilleure que la ville. Il s'agit plutôt d'une différence métaphorique, qui s'explique aussi par le mot un peu archaïque de «Lichtige Höh».

\section{Les hommes}

Des critiques contemporains, par exemple un certain ou une certaine L. Kulka, reprochent déjà à l'auteure de ne pas décrire les clients du bordel $^{23}$. Il est vrai que les hommes sont rares dans le livre. Il y a toutefois Horner, le philosophe raté, l'instructeur de $\mathrm{M}^{\mathrm{me}}$ Goldscheider et de Milada, qui est toléré au bordel dont il a fait son domicile et qui finit à l'asile d'aliénés, victime de la syphilis. Il y a aussi Gust, l'homme gâté, le grand amour de Milada, qui la déçoit cruellement; il y a enfin l'ami et ange gardien de Gust, Joszi, payé par le père pour protéger ce jeune homme fragile de toute faute irréparable. C'est lui qui met une fin à la relation entre Gust et Milada, pas seulement en lui offrant de l'argent, mais plutôt en lui révélant le caractère de Gust.

Outre ces protagonistes (une fois de plus la constellation «deux plus un », Gust représentant l'enfant), nous trouvons des policiers de la brigade des mœurs, tous corrompus, quelques trafiquants de femmes, les juifs immigrés de la tribu de la jeune Goldscheider, et, à la fin, l'avocat et l'architecte, les seuls hommes décents.

Il y a également quelques clients, ceux qui viennent avec Gust et, plus tard, ceux du salon Spizzari qui sont de bien piètre qualité. Ils ne sont pas individualisés, ce sont seulement des clients qui changent chaque jour et sont tous identiques. Il y a, dans les histoires passées des putes, des hommes un peu plus spécifiquement décrits - l'ami officier de Lollo, le fiancé de Bine, mais il est vrai que c'est plutôt un roman de femmes et pas d'hommes. Ce sont les femmes qui ont un destin, un avenir, une histoire, qui se battent et qui évoluent, pas les hommes.

23. L. Kulka, dans Neues Frauenleben, $\mathrm{n}^{0}$ 21, 1908, cahier 8, p. 216-218. 


\section{Les dialogues directs}

Les lectures critiques du roman se sont, pour la plupart, concentrées sur le contenu, sur le milieu des prostituées. J'ai essayé de démontrer qu'il est aussi bien construit, avec des oppositions et des parallèles, des mouvements vers le haut et vers le bas, structuré selon un schéma ternaire.

Il faut y ajouter les efforts linguistiques faits pour individualiser les différentes prostituées: elles sont, on l'a déjà dit, ressortissantes de différentes parties de l'Autriche-Hongrie; il faudrait une analyse linguistique plus approfondie pour décider si les différents "patois", les « fautes» de la langue parlée dominante sont le résultat de leurs langues maternelles différentes ou s'il s'agit seulement d'une tradition littéraire qui nous a appris à décoder ces types d'expression comme dénotant telle ou telle origine. La langue utilisée dans les dialogues du roman est bien réfléchie, dans le naturalisme de langue allemande, la découverte des différents patois des classes inférieures étant effectivement un concept important.

Le roman Der goldene Skarabäus désigne la vie d'une catégorie de la société qui n'a pas fait partie des canons littéraires: la vie des prostituées, du bordel, mais aussi - dans une moindre mesure - la vie des immigrés juifs. Le livre fait l'analyse de ces segments en utilisant les méthodes du marxisme, sans dédaigner les traditions littéraires établies. Pour accentuer l'origine sociale des différents personnages, il utilise beaucoup de dialogues en patois, dialecte, voire sociolecte. Il utilise aussi des sources réelles comme le procès Riehl. Ces indices permettent de considérer ce roman comme un exemple, un peu tardif, de naturalisme, même si l'issue positive et quelques parties un peu idéalistes et pathétiques n'y correspondent pas complètement ${ }^{24}$.

24. Je remercie mon amie Annie Menot pour son travail de corrections du français de mon texte. Sans elle, je n'y serais jamais arrivée. 